Article

\title{
Bioengineering of a Full-Thickness Skin Equivalent in a 96-Well Insert Format for Substance Permeation Studies and Organ-On-A-Chip Applications
}

\author{
Katharina Schimek ${ }^{1,2,{ }^{\dagger}}$, Hao-Hsiang Hsu ${ }^{3,+}{ }^{+}$, Moritz Boehme ${ }^{3}$, Jacob Jan Kornet ${ }^{3}$, Uwe Marx ${ }^{1}$, \\ Roland Lauster ${ }^{2}$, Ralf Pörtner ${ }^{3, *}$ and Gerd Lindner ${ }^{2}$ \\ 1 TissUse GmbH, D-13347 Berlin, Germany; katharina.schimek@tissuse.com (K.S.); \\ uwe.marx@tissuse.com (U.M.) \\ 2 Institute of Biotechnology, Department Medical Biotechnology, Technische Universität Berlin, \\ D-13355 Berlin, Germany; roland.lauster@tu-berlin.de (R.L.); gerd.lindner@tu-berlin.de (G.L.) \\ 3 Institute of Bioprocess and Biosystem Engineering, Hamburg University of Technology, D-21073 Hamburg, \\ Germany; hao.hsu@tuhh.de (H.-H.H.); moritz.boehme@tuhh.de (M.B.); jacob.kornet@tuhh.de (J.J.K.) \\ * Correspondence: poertner@tuhh.de; Tel.: +49-(0)40-42878-2886 \\ + These authors contributed equally to this work.
}

Received: 4 May 2018; Accepted: 1 June 2018; Published: 7 June 2018

\begin{abstract}
The human skin is involved in protecting the inner body from constant exposure to outer environmental stimuli. There is an evident need to screen for toxicity and the efficacy of drugs and cosmetics applied to the skin. To date, animal studies are still the standard method for substance testing, although they are currently controversially discussed Therefore, the multi-organ chip is an attractive alternative to replace animal testing. The two-organ chip is designed to hold 96-well cell culture inserts (CCIs). Small-sized skin equivalents are needed for this. In this study, full-thickness skin equivalents (ftSEs) were generated successfully inside 96-well CCIs. These skin equivalents developed with in vivo-like histological architecture, with normal differentiation marker expressions and proliferation rates. The 96-well CCI-based ftSEs were successfully integrated into the two-organ chip. The permeation of fluorescein sodium salt through the ftSEs was monitored during the culture. The results show a decreasing value for the permeation over time, which seems a promising method to track the development of the ftSEs. Additionally, the permeation was implemented in a computational fluid dynamics simulation, as a tool to predict results in long-term experiments. The advantage of these ftSEs is the reduced need for cells and substances, which makes them more suitable for high throughput assays.
\end{abstract}

Keywords: full thickness skin equivalents; multi-organ chip; substance permeation; 96-well cell culture insert

\section{Introduction}

The skin, the largest human organ, offers important functions. It protects the body against mechanical influences, ultraviolet light, temperature, and dehydration. In addition, the skin has sensor functions responding to touch, temperature, and pain. Apart from other biochemical and physiological functions, the skin serves as a physical barrier between the human body and the surrounding environment [1]. Understanding this barrier's function and its reactions to topically applied substances is fundamental for cosmetic and pharmacological applications. Consequently, the need to screen for the toxicity and efficacy of the skin is evident. Historically, a huge number of animal trials have been performed worldwide to address this purpose. Tests on skin irritation, for example, have been carried out using the Draize skin irritation test in rabbits, according to OECD 
TG 404 (Organization for Economic Co-operation and Development) [2]. However, there are several differences between animal and human data, for example, leading to the misclassification of chemicals using in-vivo rabbit data [3,4]. In addition, the EU Cosmetics Regulation No. 1223/2009 came into law in 2013, and prohibits the testing of cosmetic products on animals. This increases the demand for alternative methods $[5,6]$. Nowadays, human skin equivalents are widely used for animal-free tests in drug development, the chemical and cosmetics industries, and for research purposes. There are currently several skin equivalents commercially available: for example, EpiSkin ${ }^{\mathrm{TM}}$ (L'oreal, Clichy, France); EpiDerm ${ }^{\mathrm{TM}}$ SIT (MatTek Corporation, Ashland, MA, USA); SkinEthik ${ }^{\mathrm{TM}}$ RHE (SkinEthic laboratories, Lyon, France); EpiCS ${ }^{\circledR}$ (CellSystems, Troisdorf, Germany); and LabCyte EPI-MODELS24 SIT (Japan Tissue Engineering Co., Tokyo, Japan). These models are all based on only one cell type mimicking the epidermis. However, full-thickness skin equivalents (ftSEs) with an additional dermal layer are also available on the market (e.g., GraftSkin ${ }^{\circledR}$, EpiDermFT $^{\circledR}$, and Phenion ${ }^{\circledR}$ ). These models are already commonly used for substance absorption tests, in order to understand the toxicity and efficacy of pharmaceutical and cosmetic products applied to the skin $[5,7,8]$. The Franz diffusion cell system is a method very widely used to determine the absorption or permeation of a substance into the skin $[9,10]$.

A further trend for alternatives to animal substance testing, which does not focus solely on the skin, is the organ-on-a-chip system. The basic idea behind this approach is to emulate the smallest physiological unit of an organ on a chip. Thus, microenvironments must be created that correspond to the organs' natural space. The influence of substrate topography, fluidic shear stress, and culture perfusion are important factors in organ cultivation in the chip format [11,12]. Reproducing diseases on the chip to test the effectiveness of active substances is of great interest to the pharmaceutical industry. Therefore, long-term cultivation also plays an important role [13]. The variety of organ-on-a-chip-based systems is enormous. Various microfluidic perfusion culture systems have been developed on a research basis, and commercialization of these platforms keeps growing (e.g., $\mu$ Organo, ChipShop, Emulate, TissUse, Nortis, AxoSim, or Draper).

A few groups have reported skin-related cultures in chip systems [14-17]. However, looking at commercially available skin models and organ-on-a-chip systems, it becomes apparent that there is no ftSE produced in a 96-well cell culture insert (CCI) format. We have recently described a 96-well CCI-based culture within the two-organ chip (2OC) of either punched skin equivalents, commercially available in a 24-well CCI format, or native skin biopsies [18-20]. This article presents a new approach to producing ftSEs in a 96-well CCI size, which is suitable for organ-on-a-chip systems. The ftSE consists of primary human skin cells within a collagen-elastin matrix (Matriderm ${ }^{\circledR}$ ). This matrix is provided as cellular lyophilized sheets composed of bovine elastin and collagen types I, III, and V. It is used most frequently in clinics as a tissue replacement, supporting the ingrowth of cells and vessels for proper regeneration of injured skin [21].

In an already published study, a method was investigated to determine and simulate the permeation of substances in a 96-well CCI system [22,23]. Permeation is a helpful parameter that can describe the barrier properties of the skin. The diffusion coefficient can be determined with the help of a simulation, using a numerical optimization method. Here, these methods are modified for use in an organ-on-a-chip system with ftSEs for future applications. In this study, the 2OC developed by TissUse $\mathrm{GmbH}$ was used $[13,19,24-28]$. This system, about the area of a microscope slide, enables the cultivation of different organ types on a single chip. It consists of a polycarbonate adapter plate supporting the connectors for 96 -well Transwell ${ }^{\circledR}$ holders and the pump. Two culture compartments in one fluid circulation unit enable cultivation of two different tissue types. The fluid channel is made of polydimethylsiloxane (PDMS), and connects the two culture compartments supplying the organ models with nutrient solution. A micro-pump system, inspired by the human cardiovascular system [29], is operated with compressed air and enables the fluid flow. The 2OC links metabolic interaction of different organ types, thereby mimicking a part of the human body. This approach is well-established for the co-culture of human liver and skin tissue $[19,20,26,30]$. One promising aspect 
for the future application of the $2 \mathrm{OC}$ is dermal in-vitro substance testing, in combination with other tissues (e.g., liver, kidney, heart).

\section{Materials and Methods}

\subsection{Cell Isolation and Culture}

Normal human keratinocytes (NHKs) and human dermal fibroblasts (HDFs) were isolated from prepuce biopsies (EA2/091/12 of the Ethic Committee Charité University Medicine and Eth-10/15 of the Berlin Chamber of Physicians, Berlin, Germany). The prepuce was cleaned in $80 \%$ ethanol for $30 \mathrm{~s}$ and rinsed with phosphate-buffered saline (PBS) prior to isolation. The subcutaneous tissue was removed and incubated in $5 \mathrm{mg} / \mathrm{mL}$ dispase II solution (Sigma-Aldrich, St. Louis, MO, USA) at $4{ }^{\circ} \mathrm{C}$ for 15-18 $\mathrm{h}$ to separate the thin epidermal layer from the dermis.

For the isolation of NHK, the epidermis of the biopsy was cut into small pieces and incubated in trypsin/ethylenediaminetetracetic acid (EDTA) (Corning, Amsterdam, The Netherlands) for 15 min at $37^{\circ} \mathrm{C}$. The suspension was then passed through a $70 \mu \mathrm{m}$ nylon filter (BD Falcon ${ }^{\mathrm{TM}}$ Cell Strainer, Erembodegem, Belgium) and centrifuged for $5 \mathrm{~min}$ at $300 \times \mathrm{g}$. The supernatant was aspirated and the pellet re-suspended in $1 \mathrm{~mL}$ EpiLife medium $+1 \%$ human keratinocyte growth supplement (HKGS) (Gibco/Life Technologies, Darmstadt, Germany), with 1\% Penicillin/Streptomycin (Corning, Amsterdam, The Netherlands) (E1 medium). Cells were seeded at a density of $1.3 \times 10^{4} \mathrm{cells} / \mathrm{cm}^{2}$ into collagen I-coated (Biochrom GmbH, Berlin, Germany) flasks with E1 medium. The medium was changed twice a week. After a few days of culture, when cells reached $80 \%$ confluence, the NHKs and melanocytes were separated by differential trypsination. Trypsin/EDTA was added in the flask and incubated for $4-5 \mathrm{~min}$ at $37{ }^{\circ} \mathrm{C}$ to remove the melanocytes. In a second trypsination step, with an incubation time of 10-15 $\mathrm{min}$, the NHKs were passaged into new collagen-coated flasks. The NHKs were then harvested at $80 \%$ confluency, frozen in fetal calf serum (FCS) (Corning, Amsterdam, The Netherlands) with 10\% dimethyl sulfoxide (DMSO) (Carl Roth, Karlsruhe, Germany), and stored in liquid nitrogen until use.

For the isolation of HDFs, the dermis of the prepuce biopsy was cut into strips and incubated for $45 \mathrm{~min}$ in Dulbeccos's Modified Eagle Medium (DMEM) (Corning, Amsterdam, The Netherlands), containing $4 \mathrm{mg} / \mathrm{mL}$ collagenase NB 4 (Serva Electrophoresis GmbH, Heidelberg, Germany). The suspension was then passed through a $70 \mu \mathrm{m}$ nylon filter, centrifuged for $5 \mathrm{~min}$ at $300 \times g$, and the pellet was re-suspended in a $1 \mathrm{~mL}$ medium composed of DMEM, including glutamine, high glucose (Corning, Amsterdam, The Netherlands) with 1\% Penicillin/Streptomycin, and 10\% FCS (D10). Cells were seeded at $2 \times 10^{4}$ cells $/ \mathrm{cm}^{2}$ and passaged once after reaching $80 \%$ confluence. The medium was changed one day after seeding, and then every three to four days. When the HDF reached $80 \%$ confluence again, the cells were harvested, frozen in FCS with 10\% DMSO, and stored in liquid nitrogen until used.

\subsection{Manufacturing of Full Thickness Skin Equivalents}

$1 \mathrm{~mm}$ thick Matriderm ${ }^{\circledR}$ (asclepiosMedizintechnik, Gutach, Germany) pieces $4.5 \mathrm{~mm}$ in diameter were punched out and degassed in PBS (Biochrom GmbH, Berlin, Germarny) within an exsiccator (Nalgene ${ }^{\circledR}$, Rochester, NY, USA) (see Figure 1a,b). The Matriderm ${ }^{\circledR}$ punches were transferred into a 96-well, ultra-low attachment plate (Corning, Amsterdam, The Netherlands) and $200 \mu \mathrm{L}$ D10 medium supplemented with $50 \mu \mathrm{g} / \mathrm{mL}$ L-ascorbic-2-phosphate (AAP) (Sigma-Aldrich, St. Louis, MO, USA, D10-A) was added. The HDFs were harvested and a cell concentration of $0.4 \times 10^{5}$ cells $/ 50 \mu \mathrm{L}$ was adjusted. The medium was removed from the Matriderm ${ }^{\circledR}$ punches, and $50 \mu \mathrm{L}$ of the cell suspension was injected into the punches (see Figure 1c). After 15 min incubation at $37^{\circ} \mathrm{C} 150 \mu \mathrm{L}$, D10-A medium was added to the wells. The dermal equivalents (DEs) were cultivated in an incubator with a temperature of $37^{\circ} \mathrm{C}$ and a $\mathrm{CO}_{2}$ concentration of $5 \%$ for $8-11$ days, whereby the medium was exchanged three times a week (200 $\mu \mathrm{L}$ D10-A for each well). 

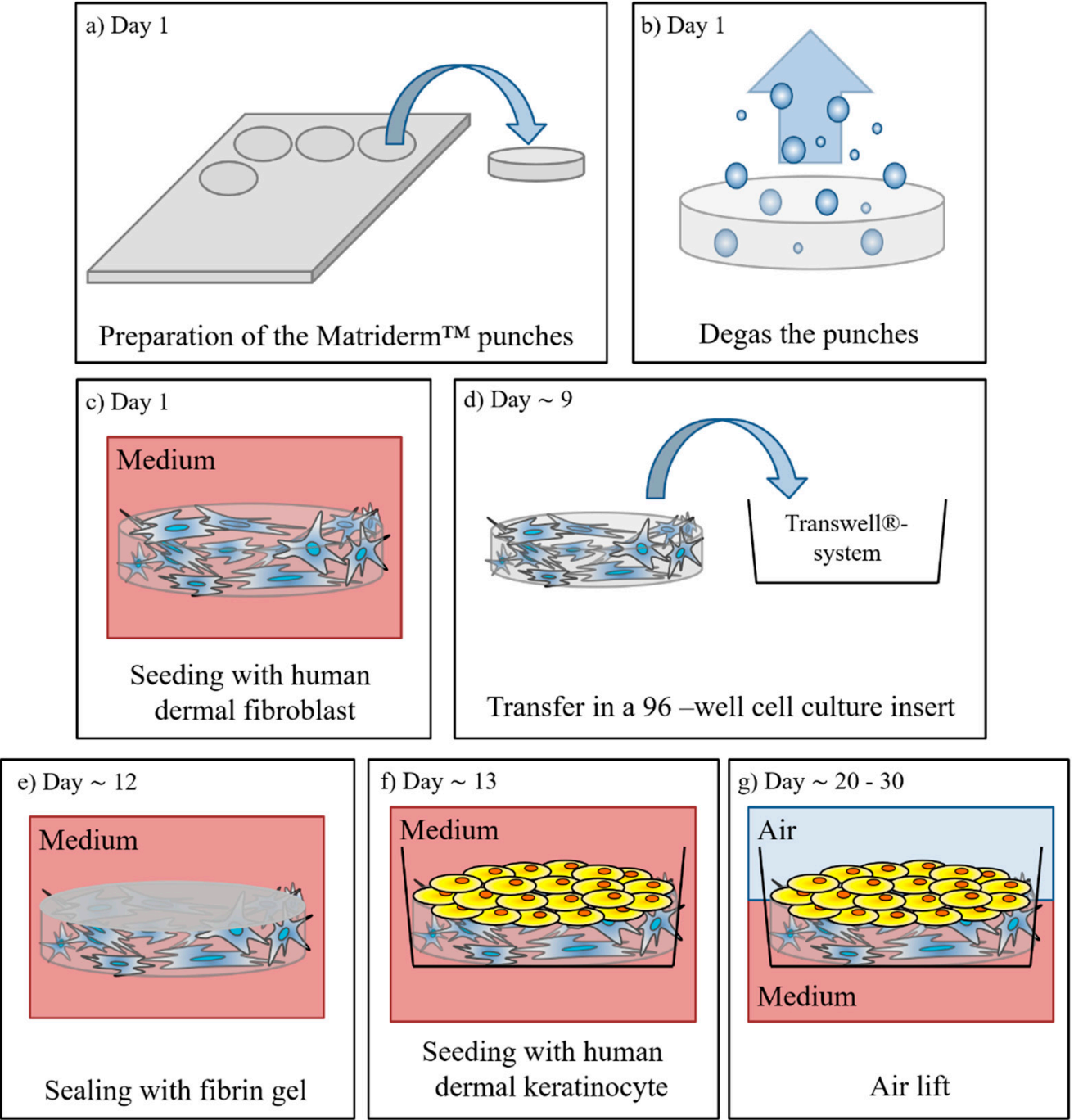

Figure 1. Schematic diagram of the ftSE cultivation. (a) Punches are cut out of the Matriderm ${ }^{\mathrm{TM}}$ matrix and (b) degassed in an exsiccator. (c) Then the matrix is seeded with human dermal fibroblasts. (d) On day nine, the $\mathrm{ftSE}$ is transferred into a cell culture insert system. (e) After four days of cultivation, the skin equivalent is sealed with a fibrin gel. (f) One day later, normal human keratinocytes are seeded onto the top of the equivalent. (g) On day 20, the equivalents are lifted to the air-liquid interface and cultivated further for $\sim 10$ days.

In the next step, the DEs were transferred into cell culture inserts (Transwells ${ }^{\circledR}$, polycarbonate membrane, $0.4 \mu \mathrm{m}$ pore size from Corning, Amsterdam, The Netherlands) (see Figure 1d); these were set in a customized polycarbonate holder and placed on a 48-well plate (Corning, Amsterdam, The Netherlands) (see Figure 2). Then, $800 \mu \mathrm{L}$ D10-A medium was added into the bottom of the well and $75 \mu \mathrm{L}$ into the cell culture insert on top of the DEs. While a standard 96-well receiver plate is normally used for the culture of reconstructed human epidermis models, ftSEs require a larger volume of medium per square centimeter culture area. Therefore, the described customized polycarbonate holder was developed. The DEs were cultivated further for 3-6 days, and the D10-A medium was changed three times a week. 

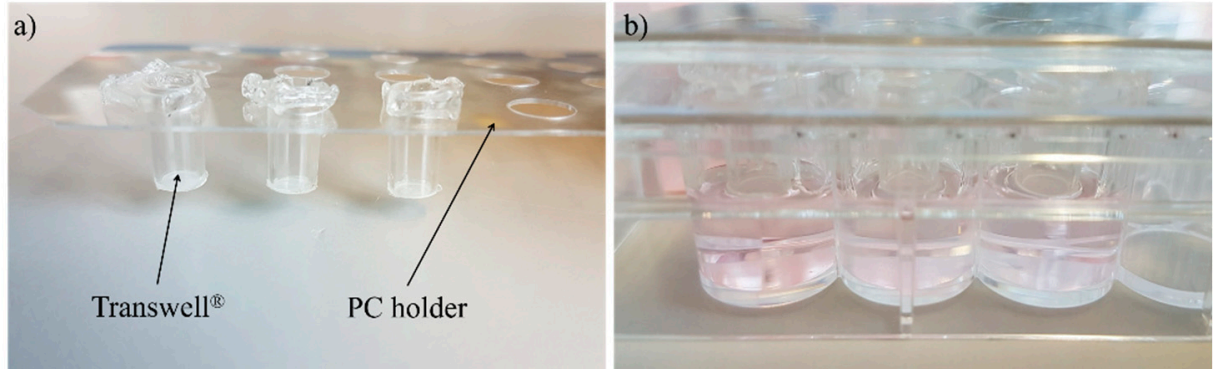

Figure 2. Pictures of the 48 -well plate Transwell ${ }^{\circledR}$ holder. (a) The Transwell ${ }^{\circledR}$ can fitted in the polycarbonate (PC) holder plate and (b) positioned in a 48-well plate.

One day before seeding the keratinocytes, the DEs were sealed to the cell culture insert by the addition of a fibrin gel solution (see Figure 1e). Fibrinogen is immediately polymerized to fibrin if thrombin is added. Therefore, two solutions were prepared and transferred to $500 \mu \mathrm{L}$ reaction tubes. A quantity of $10 \mu \mathrm{L}$ per DE of fibrinogen solution (composed of $8 \mu \mathrm{L}$ HDF $\left(0.4 \times 10^{5}\right.$ cells $/ 8 \mu \mathrm{L}$ ) solution, $1 \mu \mathrm{L}$ D10, and $1 \mu \mathrm{L}$ fibrinogen $(100 \mathrm{mg} / \mathrm{mL}$, Baxter, Vienna, Austria)) and $10 \mu \mathrm{L}$ thrombin solution (composed of $8 \mu \mathrm{L} \mathrm{CaCl}_{2}\left(40 \mathrm{mM}\right.$ in $\mathrm{H}_{2} \mathrm{O}$; Sigma-Aldrich, St. Louis, MO, USA) and $2 \mu \mathrm{L}$ thrombin ( $4 \mathrm{U} / \mathrm{mL}$; Baxter, Vienna, Austria)) was needed. The medium on top and below the DE was removed, and $10 \mu \mathrm{L}$ thrombin solution was mixed with $10 \mu \mathrm{L}$ fibrinogen solution. The mixture was transferred immediately to the DEs. The clot was incubated for $30-60 \mathrm{~min}$ at $37{ }^{\circ} \mathrm{C}$ in the $\mathrm{CO}_{2}$-incubator for polymerization. The dermis equivalents were cultured one day further in D10-A medium, as described above.

On the next day, NHKs were harvested, and a cell concentration of $0.8 \times 10^{5}$ cells $/ 50 \mu \mathrm{L}$ was adjusted with the $\mathrm{E} 1$ medium supplemented to $1.5 \mathrm{mM} \mathrm{CaCl}_{2}$ (E2). The medium was removed from the $\mathrm{ftSEs}$, and $50 \mu \mathrm{L}$ of the cell suspension was added on top (see Figure $1 \mathrm{f}$ ). After incubating for $2 \mathrm{~h}$ at $37^{\circ} \mathrm{C}, 15 \mu \mathrm{L}$ of the E2 medium were added into the top well and $900 \mu \mathrm{L}$ into the bottom well below the $\mathrm{ftSE}$. After seven days of submerged cultivation (medium changed three times a week) the medium was removed, and the ftSEs were further cultivated in an air lift (ALI) for 10 days (see Figure 1g). For the ALI cultivation, the E3 medium, composed of the E2 medium supplemented with $50 \mu \mathrm{g} / \mathrm{mL}$ AAP and $5 \mathrm{ng} / \mathrm{mL}$ keratinocyte growth factor (Sigma-Aldrich, St. Louis, MO, USA), was used $(800 \mu \mathrm{L}$ medium, in the bottom well only), and the medium was changed three times a week.

\subsection{Cultivation in Two-Organ Chip}

One part of the ftSEs were cultivated further in a 2OC, produced and provided by TissUse $\mathrm{GmbH}$ (Berlin, Germany) (see Figure 3). Further information about the 2OC can be found in Wanger et al. [20], Materne et al. [19], and Schimek et al. [25]. Prior to cultivation, the 2OC was flushed with an E3 medium. The culture compartments of the chip were both filled with $300 \mu \mathrm{L}$ of the E3 medium. Prior to integration into the chip, the Transwell ${ }^{\circledR}$ containing the $\mathrm{ftSE}$ was cut off below the bracket with a heated scalpel and removed. The Transwells ${ }^{\circledR}$ were then positioned air bubble-free into the Transwell ${ }^{\circledR}$-holder, which was screwed into one of the $2 \mathrm{OC}$ compartments. The other compartment of each circuit of a $2 \mathrm{OC}$ was used as a media reservoir, and was filled up with $600 \mu \mathrm{L}$ of the E3 medium. The 2OCs were connected to the TissUse Control Units, adjusted to a pump rate of $2 \mathrm{~Hz}$, and incubated at $37^{\circ} \mathrm{C}$ and $5 \% \mathrm{CO}_{2}$. The medium was changed every second day. 


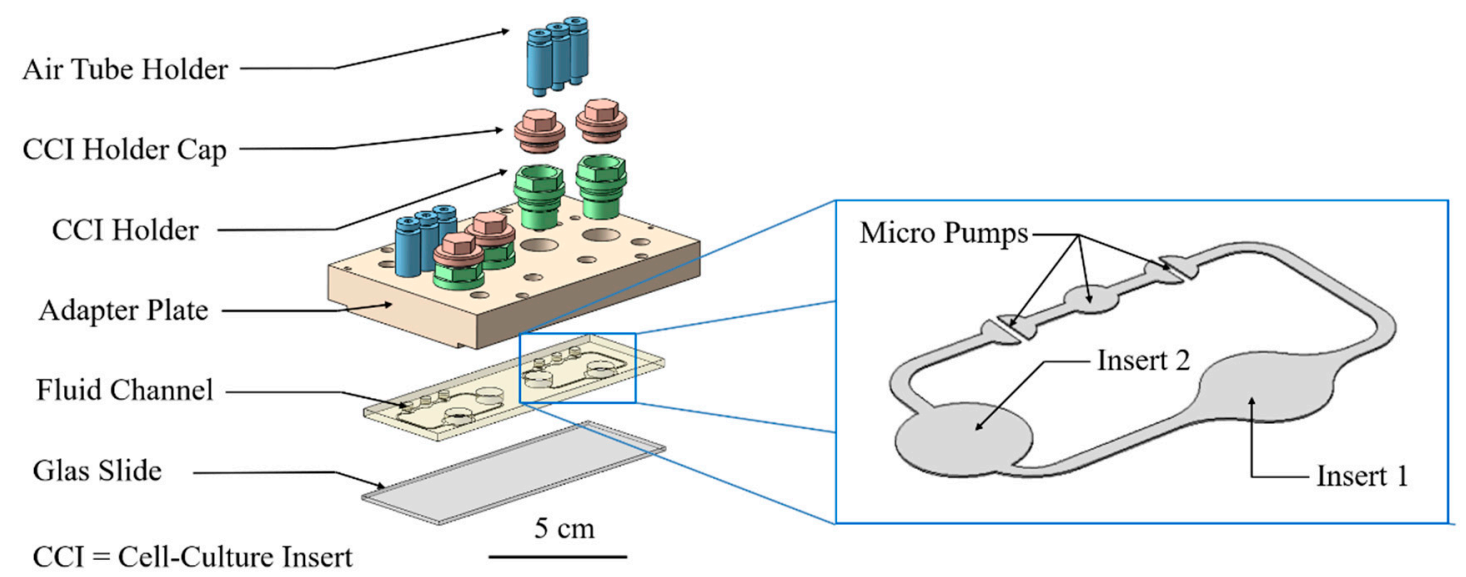

Figure 3. Exploded diagram of the Two-organ chip system. The blue box shows a zoom on the fluid channel with the position of the micro pumps, insert 1 and insert 2.

\subsection{Staining}

At the end of the culture, the tissues were harvested and frozen in cryomedium TissueTek (Sakura Finetek Germany GmbH, Staufen, Germany) and cut with a Cryotome in slices with a thickness of $8 \mu \mathrm{m}$. For the immunofluorescence staining the skin slices were fixed in acetone (Carl Roth, Karlsruhe, Germany) at $-20^{\circ} \mathrm{C}$ for $10 \mathrm{~min}$, washed with PBS and blocked with $10 \%$ goat serum (Sigma-Aldrich, St. Louis, MO, USA) in PBS for $20 \mathrm{~min}$. Tissues were then incubated with the primary antibody over night at $4{ }^{\circ} \mathrm{C}$ and washed with PBS. Subsequently, the conjugated secondary antibody and 4,6-diamidine-2-phenylindole (DAPI) (Sigma-Aldrich, St. Louis, MO, USA) (1:2000 diluted in PBS) were added and incubated for $45 \mathrm{~min}$. The sections were washed twice in PBS, covered with mounting medium and stored at $-20^{\circ} \mathrm{C}$ in the dark until imaging. The antibodies are listed on Table 1 .

Table 1. List of antibodies used for immunofluorescence staining.

\begin{tabular}{|c|c|c|}
\hline Antibodies & Dilution in PBS & Manufacturer \\
\hline Collagen I, mouse anti-human, 5.9 mg/mL & $1: 100$ & Sigma $^{1}$ \\
\hline Cytokeratin 10 , mouse anti-human, $1 \mathrm{mg} / \mathrm{mL}$ & $1: 100$ & Chemicon $^{2}$ \\
\hline Cytokeratin 15 , rabbit anti-human, $100 \mu \mathrm{g} / \mathrm{mL}$ & $1: 100$ & Chemicon $^{2}$ \\
\hline Filaggrin, mouse anti-human, $0.2 \mathrm{mg} / \mathrm{mL}$ & $1: 50$ & Thermo Fisher ${ }^{3}$ \\
\hline IgG-CF 488, goat anti-rabbit, 2 mg/mL & $1: 200$ & Biotium 4 \\
\hline IgG-CF 594, goat anti-mouse, 2 mg/mL & $1: 200$ & Biotium 4 \\
\hline Vimentin, IgG rabbit anti-human, $200 \mu \mathrm{g} / \mathrm{mL}$ & $1: 100$ & Santa Cruz ${ }^{5}$ \\
\hline
\end{tabular}

${ }^{1}$ Sigma-Aldrich, St. Louis, MO, USA. ${ }^{2}$ Merck, Darmstadt, Germany. ${ }^{3}$ Thermo Fisher, Schwerte, Germany. ${ }^{4}$ Biotium, Fremont, CA, USA. ${ }^{5}$ Santa Cruz Biotechnology, Dallas, TX, USA.

For the hematoxylin and eosin (HE) staining, the slides were fixed in acetone for $10 \mathrm{~min}$ at $-20{ }^{\circ} \mathrm{C}$ and then washed in distilled water. The slides were then stained in haematoxylin-solution (Harris, VWR, Radnor, PA, USA) for $5 \mathrm{~min}$, and washed again in distilled water. The slices were differentiated by two quick dips in $0.3 \%$ acid alcohol. The staining turned blue when tap water was run over the slides for 5 min. Staining in eosin (Merk, Darmstadt, Germany) followed for 2 min, after which the slides were washed again in tap water and then dehydrated in alcohol solutions with increasing concentration (70, 96, and 99\% and Roti ${ }^{\circledR}$-Histol (Carl Roth, Karlsruhe, Germany)) for 5 min each. The stained slides were covered with Roti-Histokitt (Carl Roth, Karlsruhe, Germany), and a cover slip was attached. 


\subsection{Permeation Measurement}

The permeation experiments were performed in 2OCs. The fluid channels of the chips were filled with E2 medium, and the ftSEs were transferred in the 2OC as described before (see Figure 4). The medium reservoir compartment was used for the acceptor, and was filled with $300 \mu \mathrm{L}$ E2 medium. Fluorescein sodium salt (Carl Roth, Karlsruhe, Germany) with a concentration of $0.1 \mathrm{mg} / \mathrm{mL}$ in E2 medium was used as donator substance, and $75 \mu \mathrm{L}$ of donator substances were applied onto the top of the $\mathrm{ftSE}$ in the CCI. The acceptor (receiver plate, bottom of the $\mathrm{ftSE}$ ) was filled with $300 \mu \mathrm{L} \mathrm{E} 2 \mathrm{medium}$ (see Figure 4). During the experiment, the $2 \mathrm{OC}$ was positioned in the incubator at $37{ }^{\circ} \mathrm{C}$ and $5 \% \mathrm{CO}^{2}$. Hourly, $100 \mu \mathrm{L}$ samples were taken from the acceptor and transferred to a 96-well plate to measure the fluorescence. After the measurement, the sample was returned into the 2OC.

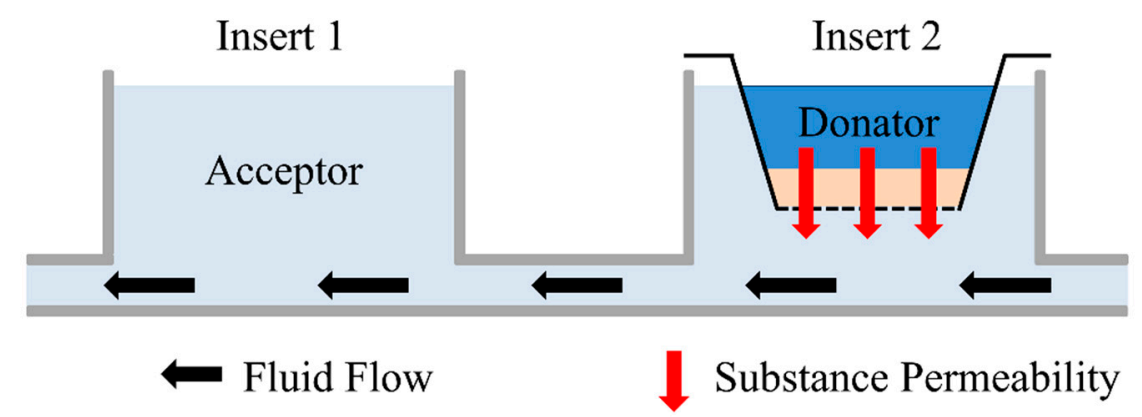

Figure 4. Schematic side view of the 2OC. The acceptor, where the samples are taken, is located at Insert 1. The 96-well CCI system with $\mathrm{ftSE}$ is positioned on Insert 2. The donator is the investigated substance on the $\mathrm{ftSE}$. Black arrows indicate the direction of the fluid flow, and the red arrows the permeation.

To determine the permeation coefficient, the following equation derived from Fick's first law was used:

$$
\frac{d c_{A}}{d t}=P \times A \frac{c_{D}}{V_{A}}
$$

where $c_{A}$ is the concentration of the substance at the acceptor, $c_{D}$ is the concentration at the donator, $t$ is time, $P$ is the permeation coefficient, $A$ is the permeation surface, and $V_{A}$ is the volume of the acceptor. This equation can only be applied when $c_{A} \gg c_{D}$.

\subsection{Numerical Simulation}

The numerical simulation was executed with the program COMSOL Multiphysics (Version 5.1, COMSOL Inc., Stockholm, Sweden) under the following assumptions:

(a) The diffusion coefficient of fluorescein sodium salt in $\mathrm{H}_{2} \mathrm{O}$ was set to a value of $1 \times 10^{-9} \mathrm{~m}^{2} / \mathrm{s}$, which is approximately $100 \times$ higher than the diffusion coefficient through the $\mathrm{ftSE}$.

(b) In the experiment, the substance diffuses through the $\mathrm{ftSE}$, and a membrane of the CCI system. This is considered to be one homogenous phase.

(c) For the computation fluid dynamic (CFD) simulation, all physical boundary effects on walls were neglected.

(d) The fluid flow in the 2OC was assumed to be a laminar. The experimentally measured mean value was used as flow velocity.

The fluid channel geometry of the 2OC was implemented in the simulation. On the position of Insert 1, a cylinder was generated with the acceptor volume. The donator and ftSE volume were also realized as a cylinder, and located at the Insert 2 position (see Figure 5). Two small cuboids were positioned on the micro pump for the in- and outflow of the fluid simulation (see Figure 5, zoomed picture). There is a gap between the cuboids that was not traversable for the flow. The volumes correspond to the real experimental setup. 


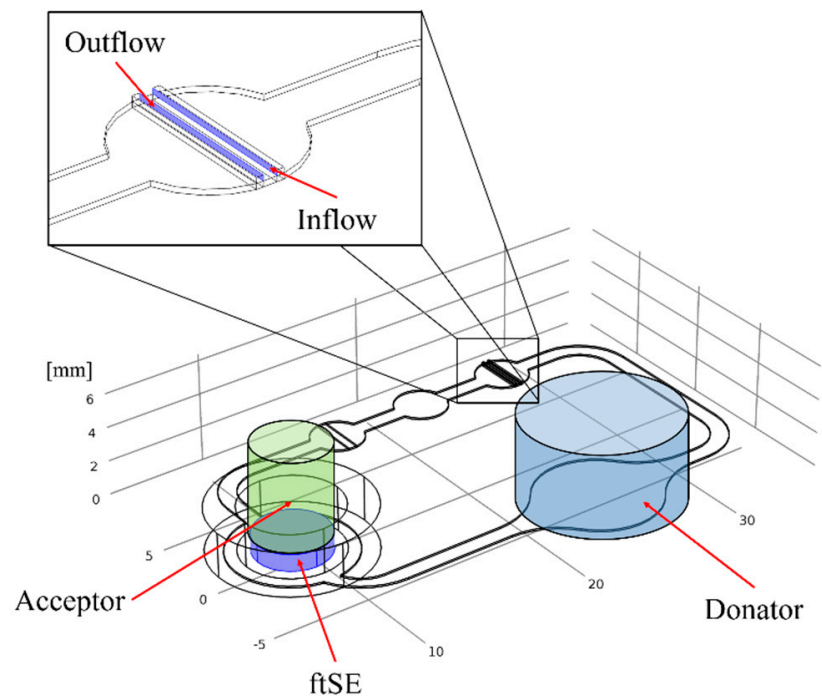

Figure 5. Geometry of the numerical simulation. The green cylinder shows the volume of the acceptor, and the blue cylinder shows the volume of the donator. At the bottom of the acceptor the ftSE is located. The zoom shows the position of the in- and outflow for the fluid simulation.

For the fluid flow simulation, the "laminar flow" module was used. All areas affected by the fluid flow were included in the CFD (exceptions are the donator, the ftSE, and the gap). The material in this area was declared as water. The position of the in- and outflow of the fluid were defined on the two small cuboids, mentioned above. The velocity was set at a value of $15.76 \mathrm{~mm} / \mathrm{s}$. A stationary study with a GMRES (generalized minimal residual method) solver was used to simulate the laminar flow. The integrated auto mash was used to generate the grid, and the element size "normal" was selected (see Figure 6).

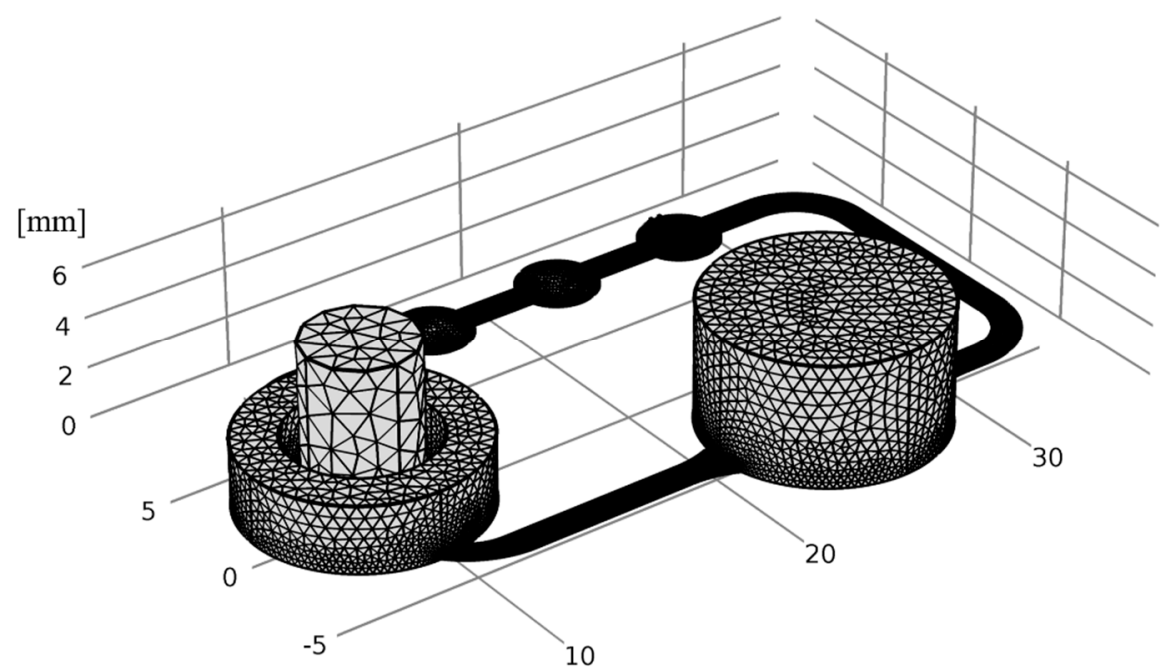

Figure 6. The mash of the numerical simulation. To generate the grit, the auto mash function with "normal" tetrahedral element size was used.

The diffusion process of fluorescein sodium salt was simulated with the "transport of diluted species" module. All areas except for the ftSE were assigned a diffusion value of $1 \times 10^{-9} \mathrm{~m}^{2} / \mathrm{s}$. The diffusion coefficient of the $\mathrm{ftSE}$ was assigned a value (calculated from the parameter adaption). All areas circulated by the liquid have been coupled to the CFD. A time-dependent study with a 
nonlinear Newton's method was used to simulate the diffusion process. The time range was set from $0 \mathrm{~s}$ to $20,000 \mathrm{~s}$, with a time step of $100 \mathrm{~s}$.

The diffusion coefficient of the ftSE was determined with the "optimization" module. For this, the experimental data were integrated in the "global last-square objective". The first column of the data was defined as the time, and the second column expresses the concentration of the donator. The concentration of the donator was set as the average concentration of the cylinder on Insert 1 , and the "global control variable" was set with an initial value of 1 and a control range between $0-1000$. For the parameter adaption, the global control variable was multiplied by the factor $1 \times 10^{-10}$. The diffusion coefficient of $\mathrm{ftSEs}$ was set as the chosen global control variable, in order to optimize the value at this area. The study of this module was implemented in the time-dependent study of the "transport od diluted species", and the SNOPT (Spares Nonlinear Optimizer) method with tolerance of $1 \times 10^{-8}$ was chosen. Exact step-by-step instructions for the simulation on an CCI system with the similar simulation modules can be found in Hsu et al. [22].

\section{Results}

\subsection{Histological Analysis of the Full-Thickness Skin Equivalents}

The cutaneous tissue morphology and expression pattern of several differentiation markers have been analyzed, using histological and immunohistochemical staining at different time points over a culture period of 24 days in the ALI, in order to characterize ftSEs over a long-term culture period. Histological staining shows that HDFs are distributed homogeneously throughout the dermis matrix after 14 days pre-culture (Figure $7 \mathrm{a}, \mathrm{g}$ ). A thin layer of fibrin glue sealed the gap between the colonized matrix and the CCI wall. As a result, an accumulation of fibroblasts formed at the upper part of the dermal equivalent (Figure $7 \mathrm{~b}, \mathrm{~h}$ ). Several epidermal layers were already apparent after seven days of ALI cultivation (Figure 7c,i). An increase in the number of layers was visible until day 10 . Furthermore, a thicker stratum corneum and an epithelium containing more keratohyalin granules in the stratum granulosum emphasized the advanced differentiation process (Figure $7 \mathrm{~d}, \mathrm{j}$ ). No differences between 10 and 17 days of ALI culture could be recognized, except for further thickening of the stratum corneum (Figure 7e,k). After 24 days, all layers were still present, but seemed to be more compact (Figure $7 f, 1)$. Keratinocytes of the basal layer were regularly arranged with a columnar structure throughout the cultivation period (Figure 7i-l).

Immunofluorescence analysis should provide an insight into the expression kinetics of the skin equivalents. Differentiation-specific keratin expression patterns are shown here by K10/K15 double staining (Figure $8 \mathrm{a}, \mathrm{e}, \mathrm{i}, \mathrm{m}, \mathrm{q}, \mathrm{u}$ ). Without any keratinocytes seeded on top of the dermis, no K10/K15 expression was observed (Figure 8a,e). At all other time points, a strict border between K15-positive basal keratinocytes and K10-positive suprabasal cells was visible (Figure 8i,m,q,u).

Filaggrin protein expression was seen during the epidermal differentiation process. This late differentiation marker is expressed and processed by cells in the stratum granulosum. Filaggrin expression in skin equivalents increased significantly over time (Figure $8 \mathrm{c}, \mathrm{g}, \mathrm{k}, \mathrm{o}, \mathrm{s}, \mathrm{w}$ ). While barely visible until seven days of air-lift culture, intense staining was seen after 17 and 24 days of culture, indicating advanced differentiation levels.

The formation of a basal membrane was proven by a prominent collagen IV staining (Figure $8 b, f, j, n, r, v)$. Collagen IV expression within the dermis and basal zone, already present after seven days of ALI culture, increased slightly over time. Deposition of collagen IV was observed, particularly near the dermal-epidermal junction, while decreasing towards the lower dermis (Figure $8 \mathrm{r}, \mathrm{v}$ ).

Vimentin was used as a marker for fibroblasts populating the dermis, which were found to be spread throughout the entire construct (Figure $8 \mathrm{~d}, \mathrm{~h}, \mathrm{l}, \mathrm{p}, \mathrm{t}, \mathrm{x}$ ). An increased number of cells accumulated within the upper part of the dermis construct. This trend continued further when keratinocytes were added on top of the dermis, resulting in a densely-populated upper dermis and a 
less-densely-populated lower dermis. This phenomenon can also be observed within the papillary and reticular dermis of native human skin in vivo.

The vitality of the skin equivalents after long-term culture was determined by TUNEL-Ki67 immuno-labelling (Figure 9). The mitotic activity of the keratinocytes, visualized by Ki67 staining, showed a still-considerable number of proliferating keratinocytes within the basal layer after 24 days of air-lift culture. Concurrently, only a few apoptotic TUNEL-positive cells were seen within the $\mathrm{ftSE}$, indicating a still viable skin equivalent. The long-term maintenance and regular differentiation characteristics suggested a balance between proliferation, differentiation, and apoptosis, leading to a physiological tissue homeostasis.
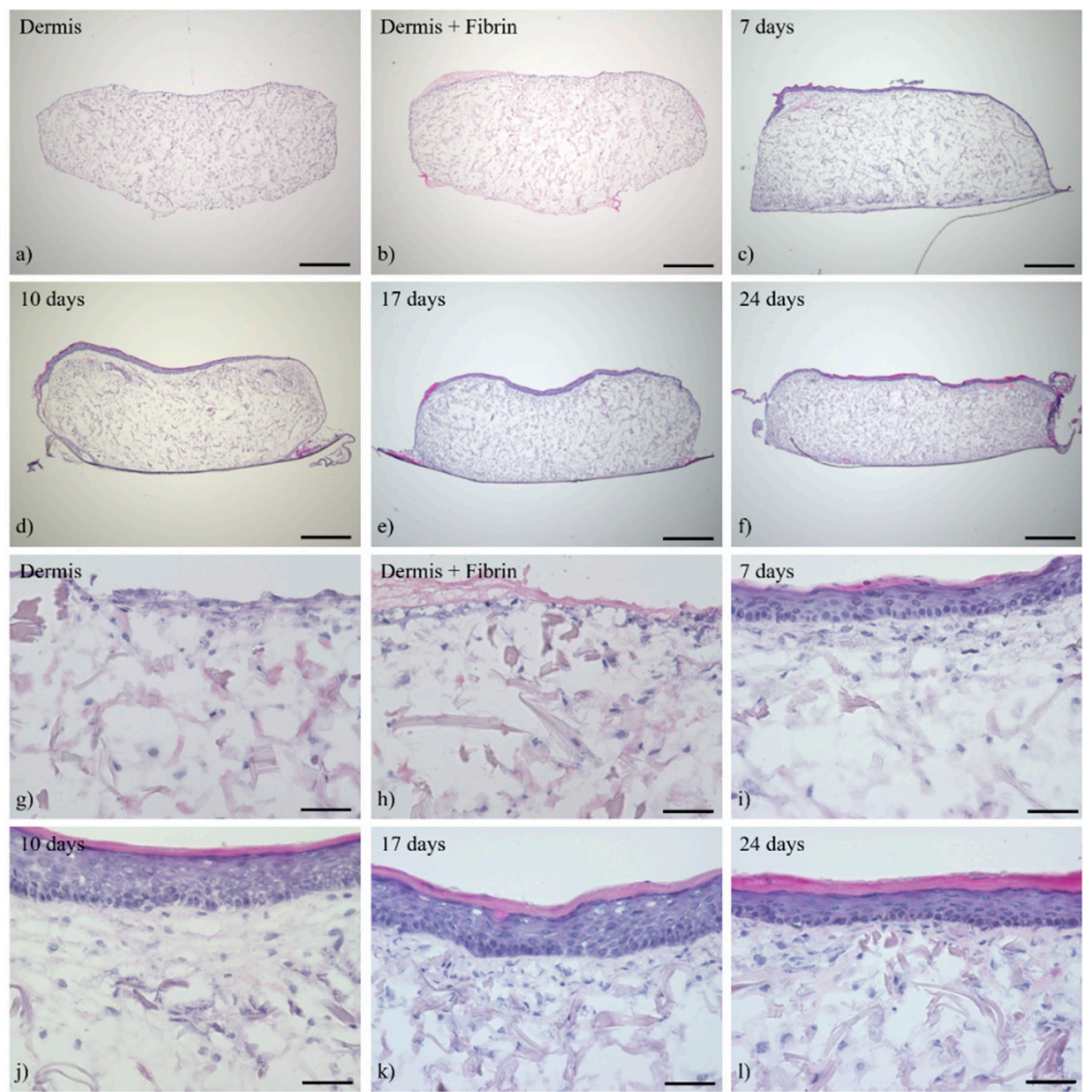

Figure 7. Hematoxylin and eosin (HE) staining of full-thickness skin equivalents (ftSEs). The ftSE morphology was evaluated at different time points. Images show representative cuts of $\mathrm{ftSEs}$ of $(\mathbf{a}, \mathbf{g})$ dermis only, $(\mathbf{b}, \mathbf{h})$ dermis with fibrin gel added on top, and (c,i) after seven days, $(\mathbf{d}, \mathbf{j}) 10$ days, $(\mathbf{e}, \mathbf{k}) 17$ days, and (f,l) 24 days of ALI culture. The skin equivalents developed a multilayered and stratified epithelium during this 24-day period. (a-f) Scale bar: $500 \mu \mathrm{m}$. (g-1) Scale bar: $50 \mu \mathrm{m}$. 

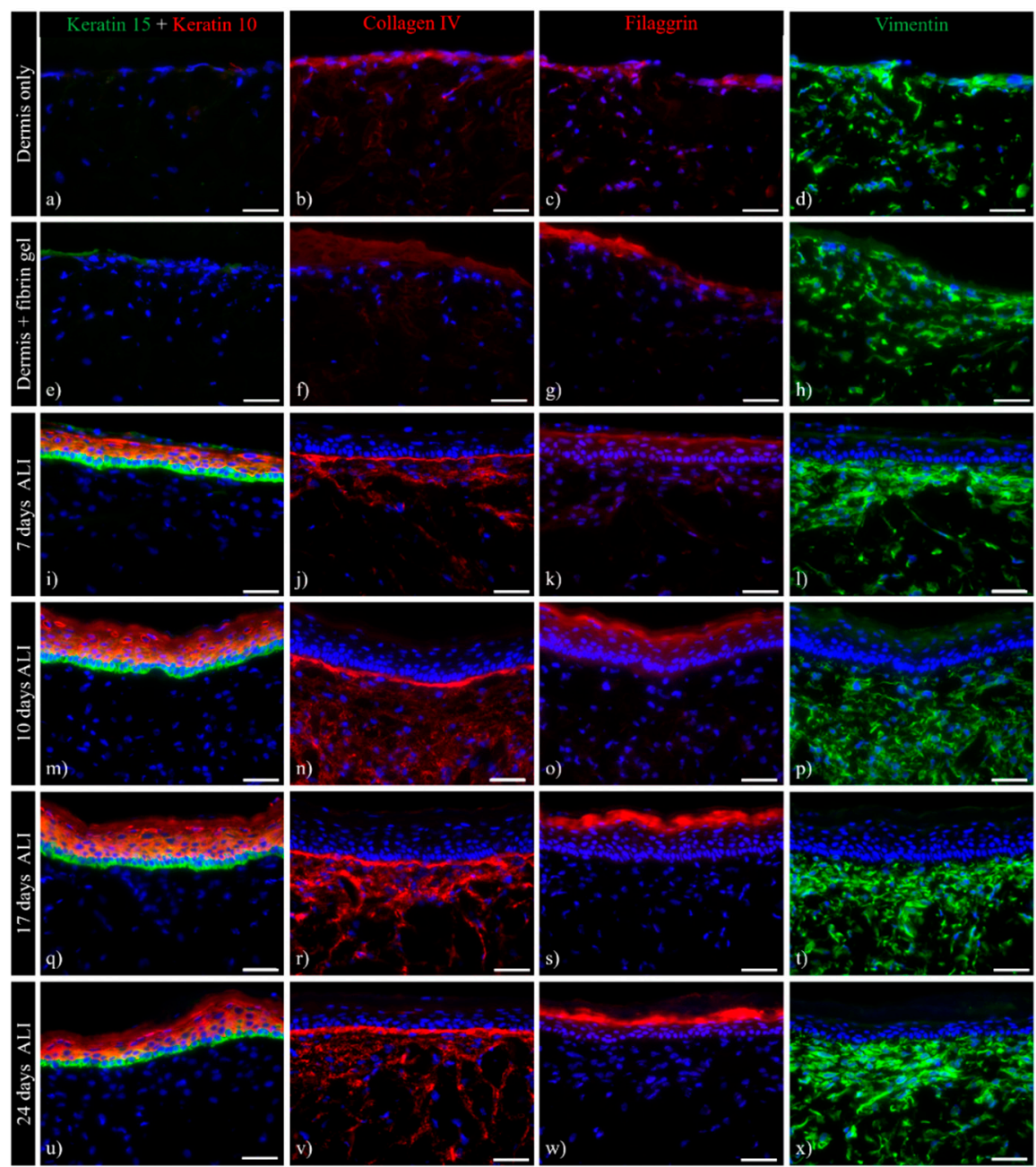

Figure 8. Immunohistochemistry of ftSEs. Expression of skin-specific markers was observed over time. Representative images of $(\mathbf{a}, \mathbf{d})$ dermis only and $(\mathbf{e}, \mathbf{f})$ dermis with added fibrin gel, as well as ftSEs after $(\mathbf{i}-\mathbf{l})$ seven days air lift (ALI), $(\mathbf{m}-\mathbf{p}) 10$ days ALI, $(\mathbf{q}-\mathbf{t}) 17$ days ALI, and $(\mathbf{u}-\mathbf{x}) 24$ days ALI were selected for further analysis. Column 1 (a,e,i,m, $\mathbf{q}, \mathbf{u})$ shows keratin 10 (red)/15 (green) double immunostaining. Column $2(\mathbf{b}, \mathbf{f}, \mathbf{j}, \mathbf{n}, \mathbf{r}, \mathbf{v})$ shows collagen IV, Column $3(\mathbf{c}, \mathbf{g}, \mathbf{k}, \mathbf{o}, \mathbf{s}, \mathbf{w})$ shows filaggrin, and Column $4(\mathbf{d}, \mathbf{h}, \mathbf{l}, \mathbf{p}, \mathbf{t}, \mathbf{x})$ shows vimentin staining. An increase in epidermal differentiation and basement membrane marker expressions at different time points indicates a physiologically relevant skin development. Counterstaining of cell nuclei with DAPI (blue). Scale bar: $50 \mu \mathrm{m}$.
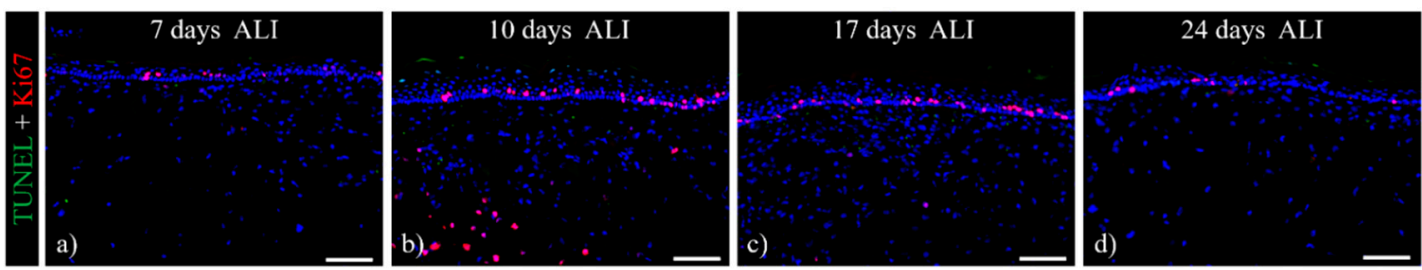

Figure 9. TUNEL-Ki67 stain of ftSEs. TUNEL-positive apoptotic cells are stained in green, and proliferative Ki67 positive cells are stained in red. A considerable number of proliferating cells within the basal layer of the ftSEs and a few apoptotic cells are found after (a) 7, (b) 10, (c) 17 and (d) 24 days of ALI cultivation. Counterstaining of cell nuclei with DAPI (blue). Scale bar: $100 \mu \mathrm{m}$. 


\subsection{Multi-Organ-Chip Culture}

Subsequently, the ftSEs were cultured for seven days in the $2 \mathrm{OC}$ to make them suitable for future co-culturing experiments with other organ equivalents.

Histological analysis of ftSEs cultivated in the 2OC showed a comparable morphology of ftSEs cultured at static conditions (Figure 10a,b).

Immunohistochemical analysis demonstrates the expression of early differentiation markers (Figure 10d) and the presence of a basement membrane (Figure 10e). The late differentiation marker filaggrin, however, is expressed only weakly (Figure 10f). The high expression of vimentin (Figure 10g) and collagen IV (Figure 10e) within the dermal matrix may reflect a higher proliferative and active level of the residing fibroblasts. The skin equivalents remained viable and proliferative, as indicated by TUNEL/Ki67 staining (Figure 10c).
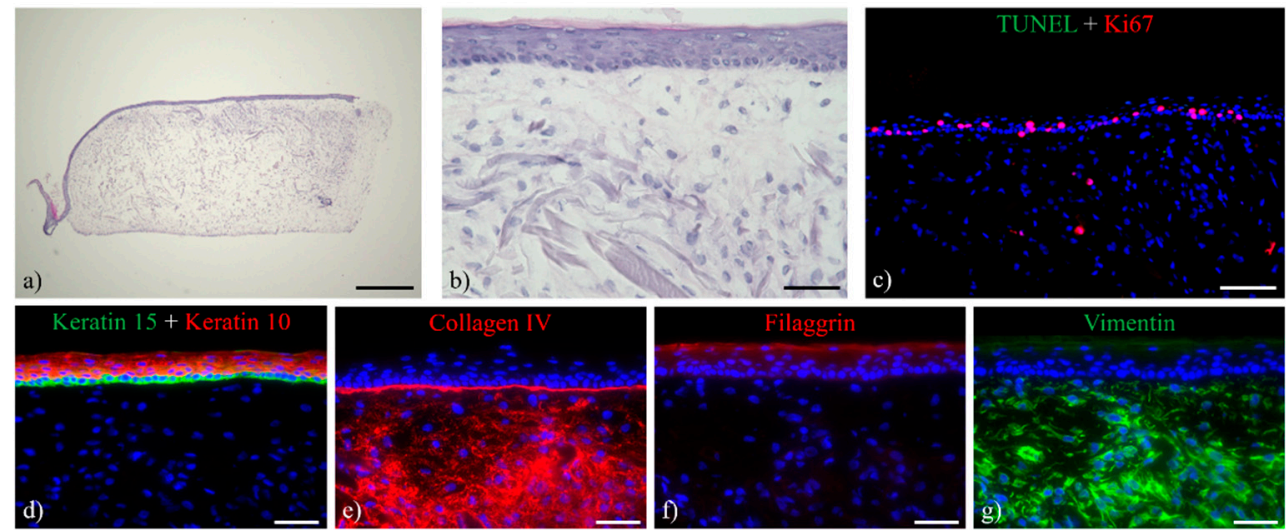

Figure 10. HE staining and immuno-labeling of ftSE cultured for seven days in the 2OC. (a,b) HE stain of the $\mathrm{ftSE}$ showed preserved morphology comprising a multilayered epidermis. (c) TUNEL-Ki67 (green and red) staining of the ftSE revealed a considerable number of proliferating cells within the basal layer of the epidermis, while only a few apoptotic cells could be detected. (d) Keratin 10 (red)/15 double stain, (e) collagen IV (red), (f) filaggrin (red), and (g) vimentin (green) staining indicate advanced levels of differentiation within the ftSE cultured in the 2OC. Counterstaining of cell nuclei with DAPI (blue).

(a) Scale bar: $500 \mu \mathrm{m}$. (b,d-g) Scale bar: $50 \mu \mathrm{m}$. (c) Scale bar: $100 \mu \mathrm{m}$.

\subsection{Trans-Epithelial Electrical Resistance and Permeation}

As skin integrity is important for substance permeation tests, the skin equivalents were evaluated macroscopically as well as by measuring the trans-epithelial electrical resistance (TEER) (Figure 11i) and permeation (Figure 12) during the entire cultivation period.

Differences in size and skin integrity could be observed macroscopically. About $40 \%$ of the skin equivalents prepared within each experiment failed due to severe shrinkage, overfloating, or other reasons. However, most of the skin equivalents remained stable until the end of the culture. The differences visible macroscopically were confirmed by differing TEER. While skin equivalents with high TEER showed a homogeneous epidermis until the CCI wall (Figure 11a-c), overfloating medium at the edges and the epidermis restricted to the center of the CCI were noticed for skin equivalents with low TEER (Figure 11e-g). This observation could be verified by HE staining (Figure 11d,h). Fewer epidermal layers could have also caused low TEER (Figure 11j,k). 

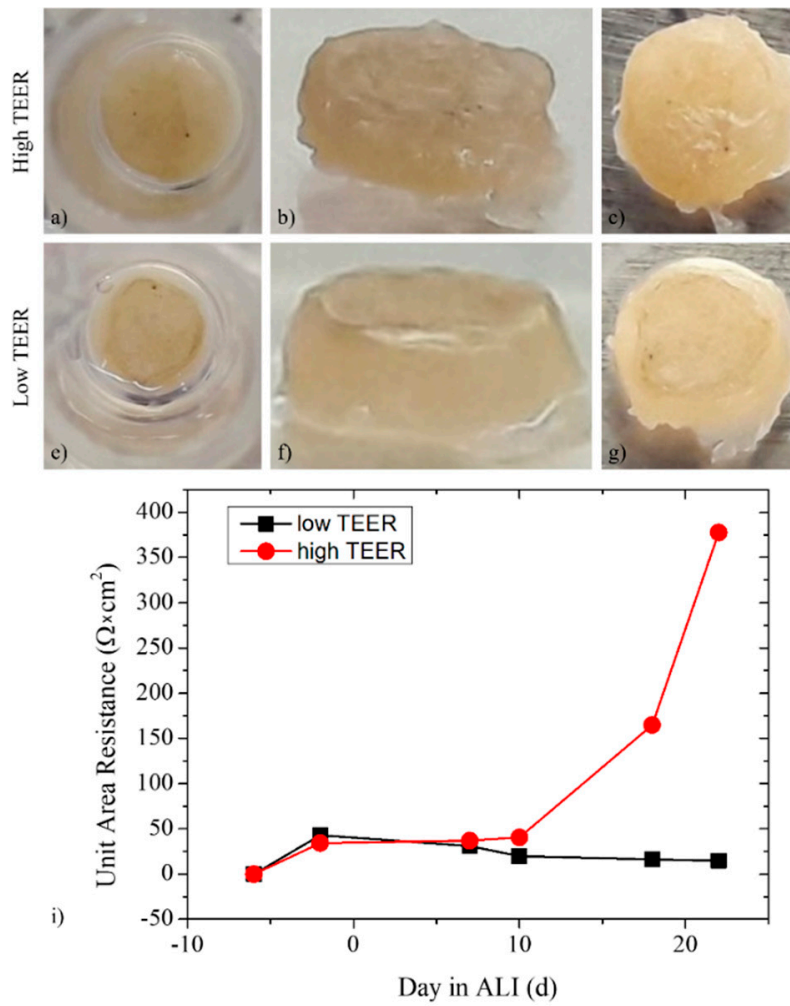
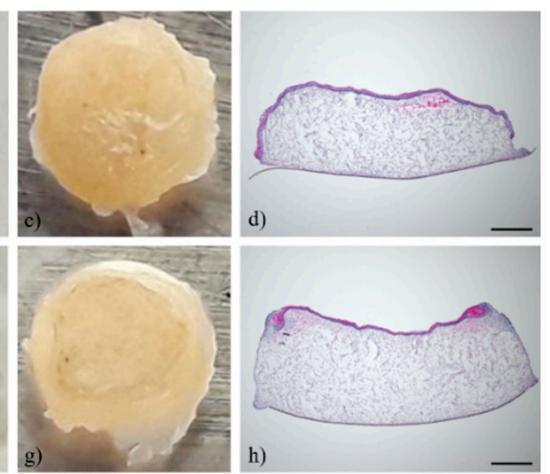

High TEER

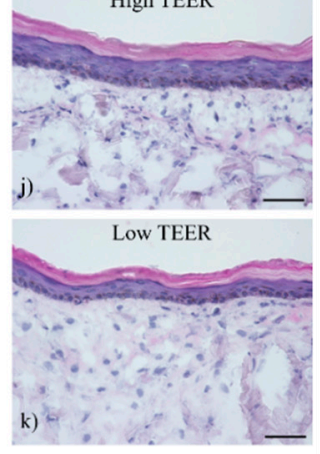

Figure 11. Macroscopic view and HE staining of the ftSE $(\mathbf{a}-\mathbf{d}, \mathbf{j})$ with high and $(\mathbf{e}-\mathbf{h}, \mathbf{k})$ low trans-epithelial electrical resistance (TEER). About $40 \%$ of the $\mathrm{ftSE}$ showed severe contraction. (i) Performance of the skin barrier was additionally evaluated by TEER. (d,h) Scale bar: $500 \mu \mathrm{m}$. (j,k) Scale bar: $50 \mu \mathrm{m}$.

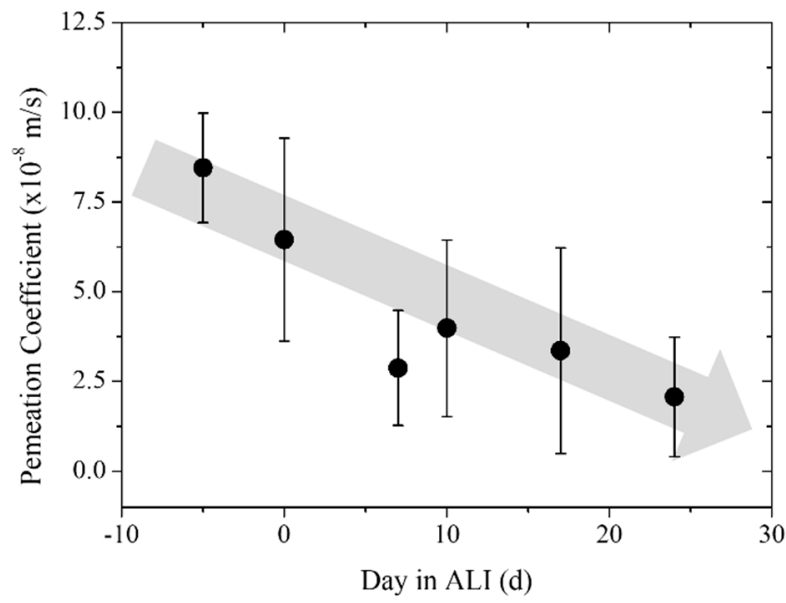

Figure 12. Permeation coefficient over the time of the $\mathrm{ftSE}$. The permeation of fluorescein sodium salt through the $\mathrm{ftSEs}$ was observed over time. The ALI was executed at day 0 . The days before pre-culture are declared with a minus. A continuous decrease of permeation over time can be seen (gray arrow).

Macroscopically defective skin equivalents were sorted out for the next experiment. The permeation of fluorescein sodium salt through the ftSEs was studied to determine a correlation with corneal differentiation. A permeation coefficient of $8.23 \times 10^{-8} \mathrm{~m} / \mathrm{s}$ was detected during the pre-culture ( 5 days before ALI). The parameter decreased to a value of $6.45 \times 10^{-8} \mathrm{~m} / \mathrm{s}$ on the day of ALI. After seven days of cultivation in ALI conditions, the coefficient was still decreasing down to a value of $2.88 \times 10^{-8} \mathrm{~m} / \mathrm{s}$, and increased slightly to a value of $3.99 \times 10^{-8} \mathrm{~m} / \mathrm{s}$ on day 10 of ALI. As the 
experiment progressed, a permeation coefficient of $3.36 \times 10^{-8} \mathrm{~m} / \mathrm{s}$ was measured on day 17 in ALI, and a coefficient of $2.07 \times 10^{-8} \mathrm{~m} / \mathrm{s}$ was measured at the end, after 24 days of cultivation. Overall, a continuous decrease of the permeation coefficient of fluorescein sodium salt through the ftSEs over the cultivation period was observed (Figure 12). This indicates a relationship between the permeation coefficient and corneal differentiation of the ftSEs. The permeation coefficients, errors, and number of samples are listed in Table 2.

Table 2. Result of the permeation experiments with fluorescein sodium salt through the ftSE. The permeation at different times during the cultivation was investigated.

\begin{tabular}{cccc}
\hline Time (Day in ALI) & Permeation Coefficient $\left(\times \mathbf{1 0}^{-\mathbf{8}} \mathbf{~} \mathbf{s}\right)$ & Error $\left(\times \mathbf{1 0}^{-\mathbf{8}} \mathbf{~} / \mathbf{s}\right)$ & Number of Samples $(\mathbf{N})$ \\
\hline Pre-culture & 8.23 & 1.53 & 3 \\
Day 0 & 6.45 & 2.83 & 4 \\
Day 7 & 2.88 & 1.60 & 5 \\
Day 10 & 3.99 & 2.46 & 5 \\
Day 17 & 3.36 & 2.86 & 5 \\
Day 24 & 2.07 & 1.66 & 5 \\
Blank & 0.03 & 0.02 & 5 \\
\hline
\end{tabular}

\subsection{Numerical Simulation}

The diffusion coefficient through the ftSE into the 2OC system was evaluated by mathematical approximation. Therefore, a numerical simulation was used to fit the diffusion coefficient with the data from the permeation experiment, described previously.

A diffusion coefficient of $20.45 \times 10^{-11} \mathrm{~m}^{2} / \mathrm{s}$ was calculated for the $\mathrm{ftSEs}$ in the pre-culture. This value decreased to a value of $4.41 \times 10^{-11} \mathrm{~m}^{2} / \mathrm{s}$ on day 0 (day of the ALI). After one week of cultivation, the diffusion coefficient was $2.55 \times 10^{-11} \mathrm{~m}^{2} / \mathrm{s}$, and increased to a value of $2.88 \times 10^{-11} \mathrm{~m}^{2} / \mathrm{s}$ on day 10. The diffusion coefficient decreased further to a value of $1.65 \times 10^{-11} \mathrm{~m}^{2} / \mathrm{s}$ on day 24 (Table 3). Similar to the permeation experiment, inversely proportional behavior was seen for the ftSEs. Validation of the simulation with the experimental data was achieved by calculating the coefficient of determination $\left(r^{2}\right)$ (Table 3). The values for the different time points are listed in Table 3. The value was highest on day 24, declining with decreasing cultivation time and reaching a value of 0.34 on day 0 . The value even fell below zero for the pre-culture. This indicates that the simulation on day 24 matches the experimental data closely, but moves away from the experimental value as it approaches pre-culture. The negative value of $r^{2}$ suggests that the mean value adjusts the experimental data better than the simulation. The relationship between the simulation and the experiment is visualized by plotting the data (Figure 13). The simulation shows the same course for the experiment on day 24. At day 0 , the concentration of the experimental data increased more rapidly compared to the simulation.

Table 3. Results of the calculated diffusion coefficient with the numerical simulation and the coefficient of determination $\left(r^{2}\right)$ regarding the experimental data. The diffusion of fluorescein sodium salt through the $\mathrm{ftSEs}$ at different cultivation times was investigated.

\begin{tabular}{ccc}
\hline Time & Diffusion Coefficient $\left(\times \mathbf{1 0}^{\mathbf{- 1 1}} \mathbf{~ m}^{\mathbf{2}} \mathbf{s}\right)$ & $\boldsymbol{r}^{\mathbf{2}}$ \\
\hline Preculter & 20.45 & -19.15 \\
Day 0 & 4.41 & 0.32 \\
Day 7 & 2.55 & $<0.01$ \\
Day 10 & 2.88 & 0.49 \\
Day 17 & 2.66 & 0.83 \\
Day 24 & 1.65 & 0.98 \\
Blank & 0.03 & 0.02 \\
\hline
\end{tabular}



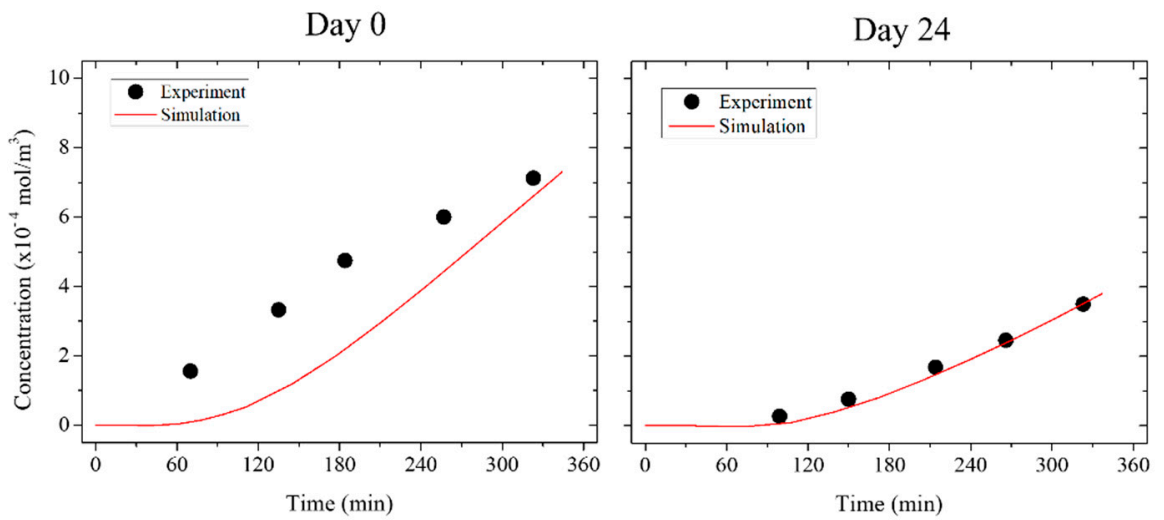

Figure 13. Comparison of the simulation with the permeation experiment. The diagrams show the donator concentration over time. The permeation of fluorescein sodium salt through the $\mathrm{ftSE}$ on day 0 (day of the ALI) and day 24 were determinant. The diffusion was calculated with numerical simulation by parameter adaption with Fick's law.

\section{Discussion}

The aim of this work was to develop an ftSE with the size of a 96-well CCI that can be used for an organ-on-a-chip system. These skin equivalents are small, so fewer cells and less substance are needed. Therefore, the $\mathrm{ftSE}$ can be used for high throughput assays.

The immunological staining showed regular epidermal development and expression of typical marker proteins. The keratinocytes alter their keratin expression with an increasing differentiation status. Undifferentiated, mitotically-active keratinocytes of the basal layer express the keratins $\mathrm{K} 5 / \mathrm{K} 14$ [31] and K15 [32]. When differentiation is initiated in the keratinocytes of the suprabasal layers, expression of $\mathrm{K} 1$ and $\mathrm{K} 10$ is characteristic [32]. Filaggrin, a protein of the cornified envelope used as a late differentiation marker [33], was found at later stages of ALI. The presence of this protein indicates a regular epidermal development of the ftSEs. Physiological paracrine signaling between keratinocytes in the epidermis and fibroblasts in the dermis is necessary for the development of the basement membrane zone. The crosstalk triggers the synthesis of a complex network of extracellular matrix located at the dermal-epidermal junction. The main components of the basement membrane zone are collagen IV, collagen VII, perlecan, nidogen, and various laminins [34]. The expression of the basement membrane protein collagen IV at the interface of the epidermis and the dermal matrix of the $\mathrm{ftSEs}$ provides evidence for their potential to organize themselves in a physiological way. Vimentin is the major intermediate filament protein of mesenchymal cells, and is used widely as a marker for dermal fibroblasts [35]. Interestingly, more fibroblasts accumulated within the upper part of the dermis. This is close to the native dermal architecture, where the fibroblasts are denser in the upper papillary dermis than in the lower reticular dermis [36]. This increased number of fibroblasts could also explain the enhanced collagen IV accumulation in the upper part of the dermis. A leak-proof connection of the skin equivalent with the CCI wall is indispensable for a controlled experiment [37]. The combination of the Matriderm ${ }^{\circledR}$ matrix with a fibrin gel to engineer the dermal component of the $\mathrm{ftSE}$ has been proven to be advantageous. The fibrin acts as a sealant, and furthermore, improves keratinocyte growth, as shown in several clinical studies [38-40].

According to OECD test guidelines (e.g., No. 428), the integrity of the skin barrier must be evaluated before a substance can be applied for penetration/absorption studies. Transepithelial/transendothelial electrical resistance is a widely accepted quantitative and noninvasive method based on measuring ohmic resistance [33]. As the intention of the ftSEs was to develop skin equivalents compatible with skin penetration/absorption studies, TEER measurements were conducted to verify the ftSEs' barrier integrity. The TEER recapitulated the findings from macroscopic and histological analyses. Shrinkage and worse epidermal performance both resulted in low TEER 
values. However, higher TEER was still low in comparison to the TEER desired described in literature. The standard limit value based on experiments with punched human skin samples, for example, was $1 \mathrm{k} \Omega$. However, these values have to be regarded with care, and vary a lot between the laboratories, as the resistance measured is dependent on the device, the frequency applied, the resulting current, the ionic strength of the solution, and the surface area of the skin sample [41]. While TEER measurements are commonly used for RHE characterization [42], they have not been described as routine tools for $\mathrm{ftSEs}$, due to severe shrinkage. One alternative is measuring the impedance across a wide spectrum of frequencies [43], or the permeation measurement of a model substance, as shown here.

Microfluidic technologies have made it possible to control the microenvironment dynamically and allow for the crosstalk of multiple organ systems, by which more reliable drug discovery studies can be conducted [44]. The aim of this study was, therefore, to include the established 96-well CCI-based skin equivalents into the 2OC, as shown previously [18-20]. Skin equivalents cultured for seven days within the $2 \mathrm{OC}$ remained viable, and showed a nearly consistent epidermal and dermal structure compared to static culture. Collagen IV and vimentin expression within the dermis increased substantially, indicating highly active fibroblasts, most likely due to the dynamic environment constantly replenishing the medium. This is in accordance with findings in other publications describing the culture of ftSEs in organ-on-a-chip systems. For example, EpidermFT ${ }^{\mathrm{TM}}$ skin equivalents (MatTek corporation, Ashland, MA, USA) cultured within the 2OC showed rearrangement and compression of the dermal matrix in dynamic cultures and a denser appearance of cell nuclei, indicating an enhanced proliferation of the fibroblasts in dynamic compared to static cultures. However, hardly any difference in viability between statically and dynamically cultured ftSEs was visible after nine days of culture, although viability was slightly worse in static cultures [18]. Research published by Abaci et al. [14] showed long-term maintenance of the dermo-epidermal ftSEs cultured on-chip that was also similar to the static culture. Contrarily, ex-vivo skin biopsies cultured within the 2OC showed substantial differences to the static culture, and the viability and morphology could be preserved over 14 days in a dynamic culture [18]. This indicates the need for perfused culture systems if skin models with increased complexity are used-for example, with integrated hair, vasculature, the immune system, or adipose tissue.

The permeation of substances was measured in a 96-well CCI system in previous experiments [22]. The permeation measurement was transferred to the $2 \mathrm{OC}$ system for further applications. The barrier function of the skin is one of its main characteristics. Thus, its permeability can be used as a quality factor to characterize the skin equivalent. It could be shown that the development of the $\mathrm{ftSE}$ can be followed with the help of the permeation measurement. It must generally be claimed that the barrier of the ftSE still differs from human skin. Permeation experiments with testosterone [8], aldosterone, hydrocortisone [45], and corticosterone [46], which have a similar molecular size as fluorescein sodium salt, show a permeation coefficient through human skin in the range of $0.4 \times 10^{-10}$ up to $3.76 \times 10^{-9} \mathrm{~m} / \mathrm{s}$. These are more than ten times smaller than the values measured for the ftSEs. However, this method of permeation determination seems to be promising for assessing the condition of the skin equivalent during the culture. This could represent a possible additional quality control for the production of the skin equivalent.

The simulation is representative of the ftSEs at the last stage of the cultivation, where the skin equivalent is well-differentiated. At the beginning of the culture, the deviation between the simulation and the experiment is high. The actual diffusion process was faster than the simulation. In all likelihood, the diffusion cannot be precisely described with Fick's law. A possible reason for this effect is the fluid flow of the 2OC. The accelerated distribution of the substance can cause anomalous diffusion [47]. The simulation has to be extended with an equation for abnormal diffusion [48] to account for this effect. 


\section{Conclusions}

In conclusion, skin equivalents were generated successfully inside 96-well CCIs, and their implementation into the $2 \mathrm{OC}$ proved its suitability as a pharmacological test system for substance penetration studies. Furthermore, it was possible to extend the simulation to the 2OC, and the diffusion coefficient could be determined for differentiated ftSEs. The numerical simulation can be used further to predict long-term effects and support and reduce experimental efforts.

Author Contributions: K.S. and H.-H.H. designed and performed the experiments, analysed and interpreted the data, and wrote the manuscript. M.B. and J.J.K. helped with the experiments and contributed to the data analysis. R.L. and U.M. developed the multi-organ-chip concept. G.L. and R.P. supervised the experimental programme and edited the manuscript.

Funding: This work was created with financial support from Deutsche Forschungsgesellschaft (DFG) under grant No. PO413/12-1 and LA 1028/7-1.

Conflicts of Interest: Uwe Marx is the CEO and shareholder and Roland Lauster and Gerd Lindner are shareholder of TissUse GmbH, a company manufacturing and commercializing the multi-organ-chip (MOC) technology. Other authors declare no conflict of interests regarding the publication of this paper.

\section{References}

1. Chuong, C.M.; Nickoloff, B.J.; Elias, P.M.; Goldsmith, L.A.; Macher, E.; Maderson, P.A.; Sundberg, J.P.; Tagami, H.; Plonka, P.M.; Therstrup-Pederson, K.; et al. What is the 'true' function of skin? Exp. Dermatol. 2002, 11, 159-187. [PubMed]

2. McNamee, P.; Hibatallah, J.; Costabel-Farkas, M.; Goebel, C.; Araki, D.; Dufour, E.; Hewitt, N.J.; Jones, P.; Kirst, A.; Le Varlet, B.; et al. A tiered approach to the use of alternatives to animal testing for the safety assessment of cosmetics: Eye irritation. Regul. Toxicol. Pharmacol. 2009, 54, 197-209. [CrossRef] [PubMed]

3. Basketter, D.; Jírova, D.; Kandárová, H. Review of skin irritation/corrosion hazards on the basis of human data: A regulatory perspective. Interdiscip. Toxicol. 2012, 5, 98-104. [CrossRef] [PubMed]

4. York, M.; Griffiths, H.A.; Whittle, E.; Basketter, D.A. Evaluation of a human patch test for the identification and classification of skin irritation potential. Contact Dermat. 1996, 34, 204-212. [CrossRef]

5. Ackermann, K.; Lombardi Borgia, S.; Korting, H.C.; Mewes, K.R.; Schäfer-Korting, M. The Phenion ${ }^{\circledR}$ full-thickness skin model for percutaneous absorption testing. Skin Pharmacol. Physiol. 2010, 23, 105-112. [CrossRef] [PubMed]

6. Asbill, C. Evaluation of a human bio-engineered skin equivalent for drug permeation studies. Pharm. Res. 2000, 17, 1092-1097. [CrossRef] [PubMed]

7. Dreher, F.; Patouillet, C.; Fouchard, F.; Zanini, M.; Messager, A.; Roguet, R.; Cottin, M.; Leclaire, J.; Benech-Kieffer, F. Improvement of the experimental setup to assess cutaneous bioavailability on human skin models: Dynamic protocol. Skin Pharmacol. Appl. Skin Physiol. 2002, 15, 31-39. [CrossRef] [PubMed]

8. Netzlaff, F.; Kaca, M.; Bock, U.; Haltner-Ukomadu, E.; Meiers, P.; Lehr, C.-M.; Schaefer, U.F. Permeability of the reconstructed human epidermis model Episkin ${ }^{\circledR}$ in comparison to various human skin preparations. Eur. J. Pharm. Biopharm. 2007, 66, 127-134. [CrossRef] [PubMed]

9. Bram, B.; Jente, B.; Christian, B.; Nathalie, R.; Filip, S.; Philipe, B.; van Jan, B.; de Bart, S. A new discriminative criterion for the development of Franz diffusion tests for transdermal pharmaceuticals. J. Pharm. Pharm. Sci. 2010, 13, 218-230.

10. Bonferoni, M.C.; Rossi, S.; Ferrari, F.; Caramella, C. A modified Franz diffusion cell for simultaneous assessment of drug release and washability of mucoadhesive gels. Pharm. Dev. Technol. 1999, 4, 45-53. [CrossRef] [PubMed]

11. Moraes, C.; Mehta, G.; Lesher-Perez, S.C.; Takayama, S. Organs-on-a-chip: A focus on compartmentalized microdevices. Ann. Biomed. Eng. 2012, 40, 1211-1227. [CrossRef] [PubMed]

12. Baker, M. Tissue models: A living system on a chip. Nature 2011, 471, 661-665. [CrossRef] [PubMed]

13. Marx, U.; Walles, H.; Hoffmann, S.; Lindner, G.; Horland, R.; Sonntag, F.; Klotzbach, U.; Sakharov, D.; Tonevitsky, A.; Lauster, R. 'Human-on-a-chip' developments: A translational cutting-edge alternative to systemic safety assessment and efficiency evaluation of substances in laboratory animals and man? Altern. Lab. Anim. 2012, 40, 235-257. [PubMed] 
14. Abaci, H.E.; Gledhill, K.; Guo, Z.; Christiano, A.M.; Shuler, M.L. Pumpless microfluidic platform for drug testing on human skin equivalents. Lab Chip 2015, 15, 882-888. [CrossRef] [PubMed]

15. Alberti, M.; Dancik, Y.; Sriram, G.; Wu, B.; Teo, Y.L.; Feng, Z.; Bigliardi-Qi, M.; Wu, R.G.; Wang, Z.P.; Bigliardi, P.L. Multi-chamber microfluidic platform for high-precision skin permeation testing. Lab Chip 2017, 17, 1625-1634. [CrossRef] [PubMed]

16. Mori, N.; Morimoto, Y.; Takeuchi, S. Skin integrated with perfusable vascular channels on a chip. Biomaterials 2017, 116, 48-56. [CrossRef] [PubMed]

17. Sriram, G.; Alberti, M.; Dancik, Y.; Wu, B.; Wu, R.; Feng, Z.; Ramasamy, S.; Bigliardi, P.L.; Bigliardi-Qi, M.; Wang, Z. Full-thickness human skin-on-chip with enhanced epidermal morphogenesis and barrier function. Mater. Today 2018, 21, 326-340. [CrossRef]

18. Ataç, B.; Wagner, I.; Horland, R.; Lauster, R.; Marx, U.; Tonevitsky, A.G.; Azar, R.P.; Lindner, G. Skin and hair on-a-chip: In vitro skin models versus ex vivo tissue maintenance with dynamic perfusion. Lab Chip 2013, 13, 3555-3561. [CrossRef] [PubMed]

19. Materne, E.-M.; Maschmeyer, I.; Lorenz, A.K.; Horland, R.; Schimek, K.M.S.; Busek, M.; Sonntag, F.; Lauster, R.; Marx, U. The Multi-organ chip-A microfluidic platform for long-term multi-tissue coculture. J. Vis. Exp. 2015. [CrossRef] [PubMed]

20. Wagner, I.; Materne, E.-M.; Brincker, S.; Süßbier, U.; Frädrich, C.; Busek, M.; Sonntag, F.; Sakharov, D.A.; Trushkin, E.V.; Tonevitsky, A.G.; et al. A dynamic multi-organ-chip for long-term cultivation and substance testing proven by 3D human liver and skin tissue co-culture. Lab Chip 2013, 13, 3538-3547. [CrossRef] [PubMed]

21. Keck, M.; Haluza, D.; Burjak, S.; Eisenbock, B.; Kamolz, L.-P.; Frey, M. Cultivation of keratinocytes and preadipocytes on a collagen-elastin scaffold (Matriderm ${ }^{\circledR}$ ): First results of an in vitro study. Eur. Surg. 2009, 41, 189-193. [CrossRef]

22. Hsu, H.-H.; Kracht, J.-K.; Harder, L.E.; Rudnik, K.; Lindner, G.; Schimek, K.; Marx, U.; Pörtner, R. A method for determination and simulation of permeability and diffusion in a 3D tissue model in a membrane insert system for multi-well plates. J. Vis. Exp. 2018. [CrossRef] [PubMed]

23. Hsu, H.-H.; Schimek, K.; Marx, U.; Pörtner, R. Measurement and simulation of permeation and diffusion in native and cultivated tissue constructs. In Biomaterials in Regenerative Medicine; Dobrzański, L.A., Ed.; IntechOpen: London, UK, 2018.

24. Marx, U.; Atac, B.; Brincker, S.; Busek, M.; Drewell, C.; Eger, R.; Fischer, M.; Frädrich, C.; Geßner, D.; Hasenberg, T.; et al. Multi-Organ-Chips: Developing a Human Chip-Based Plattform for Repeated Does Toxicity Testing; Technische Universität Berlin: Berlin, Germany, 2012.

25. Schimek, K.; Busek, M.; Brincker, S.; Groth, B.; Hoffmann, S.; Lauster, R.; Lindner, G.; Lorenz, A.; Menzel, U.; Sonntag, F.; et al. Integrating biological vasculature into a multi-organ-chip microsystem. Lab Chip 2013, 13, 3588-3595. [CrossRef] [PubMed]

26. Materne, E.-M.; Ramme, A.P.; Terrasso, A.P.; Serra, M.; Alves, P.M.; Brito, C.; Sakharov, D.A.; Tonevitsky, A.G.; Lauster, R.; Marx, U. A multi-organ chip co-culture of neurospheres and liver equivalents for long-term substance testing. J. Biotechnol. 2015, 205, 36-46. [CrossRef] [PubMed]

27. Materne, E.-M.; Tonevitsky, A.G.; Marx, U. Chip-based liver equivalents for toxicity testing-Organotypicalness vs. cost-efficient high throughput. Lab Chip 2013, 13, 3481-3495. [CrossRef] [PubMed]

28. Sonntag, F.; Gruchow, M.; Wagner, I.; Lindner, G.; Marx, U. Miniaturisierte humane organtypische Zell-und Gewebekulturen. Biospektrum 2011, 17, 418-421. [CrossRef]

29. Busek, M.; Nötzel, M.; Polk, C.; Sonntag, F. Characterization and simulation of peristaltic micropumps. J. Sens. Sens. Syst. 2013, 2, 165-169. [CrossRef]

30. Maschmeyer, I.; Hasenberg, T.; Jaenicke, A.; Lindner, M.; Lorenz, A.K.; Zech, J.; Garbe, L.-A.; Sonntag, F.; Hayden, P.; Ayehunie, S.; et al. Chip-based human liver-intestine and liver-skin co-cultures-A first step toward systemic repeated dose substance testing in vitro. Eur. J. Pharm. Biopharm. 2015, 95, 77-87. [CrossRef] [PubMed]

31. Fuchs, E.; Green, H. Changes in keratin gene expression during terminal differentiation of the keratinocyte. Cell 1980, 19, 1033-1042. [CrossRef]

32. Bose, A.; Teh, M.-T.; Mackenzie, I.C.; Waseem, A. Keratin k15 as a biomarker of epidermal stem cells. Int. J. Mol. Sci. 2013, 14, 19385-19398. [CrossRef] [PubMed] 
33. Sandilands, A.; Sutherland, C.; Irvine, A.D.; McLean, W.H.I. Filaggrin in the frontline: Role in skin barrier function and disease. J. Cell Sci. 2009, 122, 1285-1294. [CrossRef] [PubMed]

34. Breitkreutz, D.; Koxholt, I.; Thiemann, K.; Nischt, R. Skin basement membrane: The foundation of epidermal integrity-BM functions and diverse roles of bridging molecules nidogen and perlecan. BioMed Res. Int. 2013, 2013, 179784. [CrossRef] [PubMed]

35. Driskell, R.R.; Lichtenberger, B.M.; Hoste, E.; Kretzschmar, K.; Simons, B.D.; Charalambous, M.; Ferron, S.R.; Herault, Y.; Pavlovic, G.; Ferguson-Smith, A.C.; et al. Distinct fibroblast lineages determine dermal architecture in skin development and repair. Nature 2013, 504, 277-281. [CrossRef] [PubMed]

36. Sorrell, J.M.; Baber, M.A.; Caplan, A.I. Clonal characterization of fibroblasts in the superficial layer of the adult human dermis. Cell Tissue Res. 2007, 327, 499-510. [CrossRef] [PubMed]

37. Groeber, F.; Holeiter, M.; Hampel, M.; Hinderer, S.; Schenke-Layland, K. Skin tissue engineering-In vivo and in vitro applications. Adv. Drug Deliv. Rev. 2011, 63, 352-366. [CrossRef] [PubMed]

38. Horch, R. Single-cell suspensions of cultured human keratinocytes in fibrin-glue reconstitute the epidermis. Cell Transpl. 1998, 7, 309-317. [CrossRef]

39. Pellegrini, G.; Ranno, R.; Stracuzzi, G.; Bondanza, S.; Guerra, L.; Zambruno, G.; Micali, G.; de Luca, M. The control of epidermal stem cells (holoclones) in the treatment of massive full-thickness burns with autologous keratinocytes cultured on fibrin. Transplantation 1999, 68, 868-879. [CrossRef] [PubMed]

40. Ronfard, V.; Rives, J.M.; Neveux, Y.; Carsin, H.; Barrandon, Y. Long-term regeneration of human epidermis on third degree burns transplanted with autologous cultured epithelium grown on a fibrin matrix. Transplantation 2000, 70, 1588-1598. [CrossRef] [PubMed]

41. Guth, K.; Schäfer-Korting, M.; Fabian, E.; Landsiedel, R.; van Ravenzwaay, B. Suitability of skin integrity tests for dermal absorption studies in vitro. Toxicol. Vitro 2015, 29, 113-123. [CrossRef] [PubMed]

42. Netzlaff, F.; Lehr, C.-M.; Wertz, P.W.; Schaefer, U.F. The human epidermis models EpiSkin, SkinEthic and EpiDerm: An evaluation of morphology and their suitability for testing phototoxicity, irritancy, corrosivity, and substance transport. Eur. J. Pharm. Biopharm. 2005, 60, 167-178. [CrossRef] [PubMed]

43. Groeber, F.; Engelhardt, L.; Egger, S.; Werthmann, H.; Monaghan, M.; Walles, H.; Hansmann, J. Impedance spectroscopy for the non-destructive evaluation of in vitro epidermal models. Pharm Res. 2015, 32, 1845-1854. [CrossRef] [PubMed]

44. Hasan, A.; Paul, A.; Vrana, N.E.; Zhao, X.; Memic, A.; Hwang, Y.-S.; Dokmeci, M.R.; Khademhosseini, A. Microfluidic techniques for development of 3D vascularized tissue. Biomaterials 2014, 35, 7308-7325. [CrossRef] [PubMed]

45. Johnson, M.E.; Blankschtein, D.; Langer, R. Evaluation of solute permeation through the stratum corneum: Lateral bilayer diffusion as the primary transport mechanism. J. Pharm. Sci. 1997, 86, 1162-1172. [CrossRef] [PubMed]

46. Mitragotri, S.; Edwards, D.A.; Blankschtein, D.; Langer, R. A mechanistic study of ultrasonically-enhanced transdermal drug delivery. J. Pharm. Sci. 1995, 84, 697-706. [CrossRef] [PubMed]

47. Metzler, R.; Klafter, J. The random walk's guide to anomalous diffusion: A fractional dynamics approach. Phys. Rep. 2000, 339, 1-77. [CrossRef]

48. Saxton, M.J. Anomalous subdiffusion in fluorescence photobleaching recovery: A monte carlo study. Biophys. J. 2001, 81, 2226-2240. [CrossRef]

(C) 2018 by the authors. Licensee MDPI, Basel, Switzerland. This article is an open access article distributed under the terms and conditions of the Creative Commons Attribution (CC BY) license (http://creativecommons.org/licenses/by/4.0/). 